\title{
Smoking status informs about the risk of advanced serrated polyps in a screening population
}

Authors

Institutions
J. E. G. IJspeert ${ }^{1}$, P. M. Bossuyt ${ }^{2}$, E. J. Kuipers ${ }^{3}$, I. Stegeman ${ }^{4}$, T. R. de Wijkerslooth ${ }^{1}$, E. M. Stoop ${ }^{3}$, M. E. van Leerdam ${ }^{5}$, E. Dekker ${ }^{1}$

Institutions are listed at the end of article.
Bibliography

DOI http://dx.doi.org/

10.1055/s-0034-1393361

Published online: 27.11.2015

Endoscopy International Open

2016; 04: E73-E78

(c) Georg Thieme Verlag KG

Stuttgart $\cdot$ New York

E-ISSN 2196-9736

\section{Corresponding author}

Evelien Dekker, MD PhD

Department of

Gastroenterology and

Hepatology

Academic Medical Centre

Meibergdreef 91105 AZ

Amsterdam

The Netherlands

Phone: +3120566 4702

Fax: +31 206917033

e.dekker@amc.uva.nl
Background and study aims: Evidence has accumulated that approximately $15 \%$ to $30 \%$ of colorectal cancers (CRC) arise from serrated polyps (SP). Population screening, therefore, should be designated to detect advanced SP, in addition to advanced adenomas and CRC. We aimed to evaluate whether CRC risk factors also act as risk factors for advanced SP.

Patients and methods: Data were collected in the colonoscopy arm of a multicenter randomized trial comparing colonoscopy with CT-colonography for primary population screening. Information on risk factors was obtained by screening participants before colonoscopy with a validated risk questionnaire. Advanced SP were defined as $\mathrm{SP} \geq 10 \mathrm{~mm}$ and/or with dysplasia. Endoscopists were instructed to resect all detected lesions. Odds ratios (OR) for the detection of advanced SP as most advanced lesion were calculated using multiple logistic regression analysis.

\section{Introduction \\ $\nabla$}

Endoscopic detection and resection of colorectal lesions can decrease colorectal cancer mortality $[1,2]$. Due to the high prevalence of disease, slow progression from polyp to invasive cancer, and the possibility to resect its precursor lesions, population-based screening for colorectal cancer has been shown successful and cost-effective in reducing cancer mortality [3-5].

Biennial screening with a fecal immunochemical test (FIT), followed by a colonoscopy in case of a positive FIT, has been adopted in several European countries [6,7]. Although suitable for population screening, the diagnostic accuracy of FITbased screening is far from perfect.[8] Several adjustments for the pre-selection of high-risk participants in add to FIT-only testing, therefore, have been suggested $[9,10]$.
Results: Of 6600 invited participants, 1426 underwent a colonoscopy and 1236 also completed the questionnaire. In 40 participants an advanced SP was the most advanced lesion detected. Multivariate analysis demonstrated a strong association between current smoking and the presence of at least one advanced SP (OR 4.50; 95\% Cl $2.23-8.89 ; P<0.001)$. A significant association was also demonstrated for higher fiber intake (OR 1.36 per 20 gram intake; CI 1.07-1.73; $P=$ 0.01 ). Other clinical CRC risk factors did not show a significant association with the presence of at least one advanced SP in the univariate analyses. Fecal haemoglobin levels were also not significantly associated with the presence of advanced SPs (OR 1.00 per $10 \mathrm{ng} / \mathrm{mL}$ CI $0.97-1.03, P=0.99$ ). Conclusions: Current smoking is a strong clinical risk factor for the presence of advanced SPs. As such, smoking status could contribute to risk stratification in targeted CRC population screening. Dutch Trial Register: NTR1829 (www.trialregister. nl)

Research from the last decade showed that not just adenomas but also a subset of serrated polyps can progress to colorectal cancer. The World Health Organization classifies serrated polyps into the subgroups hyperplastic polyps, sessile serrated adenomas/polyps without dysplasia, sessile serrated adenomas/polyps with dysplasia, and traditional serrated adenomas [11]. Diminutive and/or small hyperplastic polyps located in the rectosigmoid are generally considered benign, whereas larger and/or proximally located hyperplastic polyps, sessile serrated adenomas/polyps, and traditional serrated adenomas are considered to possess a higher neoplastic potential $[12,13]$. The latter do so via the alternative serrated neoplasia pathway $[12,14]$. These serrated polyps may be held responsible in the development of a relatively large amount of interval cancers [15, 16]. Based on these considerations, the European 
Society for Gastrointestinal Endoscopy has recommended that people with large $(\geq 10 \mathrm{~mm})$ or dysplastic serrated polyps (referred to as advanced serrated polyps) should be classified as at high risk of developing colorectal cancer [17]. Accordingly, it has been suggested that population-based screening should not only aim to detect participants with advanced neoplasia, defined as advanced adenomas or colorectal cancer, but also those with large serrated polyps [10].

Expanding the target lesions and the definition of people at risk will potentially have a major impact on the performance of the different methods of colorectal cancer screening. A recent study showed that FIT had no value in the detection of serrated polyps in a screening setting, whereas stool DNA testing might have this diagnostic potential [18]. However, DNA testing is expensive and its diagnostic performance does not seem seems good enough yet to accurately detect advanced serrated polyps. Clinical risk factors for advanced serrated polyps may offer a noninvasive and inexpensive means of detection.

The aim of this study was to evaluate whether the well-known clinical risk factors for the detection of advanced adenomas and CRC, such as smoking, also act as risk factors for advanced serrated polyps.

\section{Patients and methods}

$\nabla$

\section{Study design}

We analyzed data collected in the colonoscopy arm of a multicenter randomized trial conducted in the Netherlands from June 2009 until July 2010, comparing colonoscopy with computed tomography (CT) colonography for primary population screening (COCOS). In 2010, population screening for CRC had not yet been introduced in the Netherlands. Detailed information about the study protocol has been described previously [19]. The trial was registered in the Dutch Trial Register: NTR1829 (www.trialregister.nl). Ethical approval was obtained from the Dutch National Health Council. (2009/03WBO, The Hague, The Netherlands)

\section{Study population}

A total of 6600 asymptomatic individuals from the greater Amsterdam and Rotterdam regions were invited to undergo colonoscopy as primary CRC screening. Individuals who had undergone a complete colonic examination (colonoscopy, CT colonography and/or double contrast barium enema) within the 5 years prior to the invitation were excluded from participation in the trial. Individuals who were already under colonoscopic surveillance (e.g., personal history of CRC, adenomas or inflammatory bowel disease) and/or had end-stage disease and a life expectancy $<5$ years were also excluded.

\section{Fecal immunochemical testing}

Participants willing to undergo a colonoscopy were invited to complete a one-sample FIT (OC-Sensor, Eiken Chemical CO., LTD., Japan) prior to the colonoscopy. Informed consent was obtained from all individuals who agreed to participate. All participants were verbally instructed how to properly perform the FIT; they could do so either at home or in one of the screening centers. Participants were instructed to perform the FIT at home within 48 hours before the colonoscopy, but before the start of their bowel preparation, and to bring the test to the screening center on the day of colonoscopy. As a second option, participants could call their screening center directly after performing the FIT, so that the test could be collected at home within 48 hours. FIT samples were immediately stored at $-20^{\circ} \mathrm{C}$ and analysed within 6 weeks. Detailed information about this procedure has been previously described [8].

\section{Risk questionnaire}

Participants willing to undergo colonoscopy were also invited to complete a questionnaire with questions about a set of putative CRC risk factors. These risk factors were selected based on a review of the existing literature [20]. Only those variables that could be obtained without additional testing were eligible for the questionnaire. The questionnaire consisted of 10 independent items; it was developed based on three pre-existing validated risk questionnaires: the Prevention Compass, the Municipal Health Agency, and the Interheart questionnaire [21 - 23]. Selected risk factors were: gender, age, familial history of CRC, adiposity, smoking behavior, sleeping behavior, amount of exercise, alcohol consumption, fiber intake, calcium intake, red meat consumption and aspirin use. Detailed information about the risk factor selection procedure and development of the risk questionnaire is available elsewhere [20]. The risk questionnaire was handed out to the participants at the day of the screening colonoscopy and collected directly before this procedure.

\section{Colonoscopy}

All colonoscopies were performed in one of the two participating academic screening centers by highly experienced gastroenterologists, each having performed more than 1000 colonoscopies at the beginning of the trial. Colonoscopists were blinded to the results of the FIT as well as to response to the risk questionnaire. All colonoscopies were performed according to the standard quality indicators defined by the American Society for Gastrointestinal Endoscopy and recorded on DVD [24]. Pre-colonoscopy bowel cleansing was obtained by a low-fiber diet, $2 \mathrm{~L}$ of hypertonic polyethylene glycol solution (Moviprep; Norgine bv, Amsterdam, the Netherlands) and $2 \mathrm{~L}$ of clear fluids. Colonoscopy was performed under conscious sedation using intravenous midazolam (Dormicum, Actavis, Baarn, the Netherlands) and fentanyl (Bipharma, Weesp, the Netherlands), at the discretion of both the participant as well as the endoscopist. Cecal intubation was confirmed by still images of the ileocecal valve as well as the appendiceal orifice or by intubation of the terminal ileum. Quality of bowel preparation was measured using the validated Ottawa bowel preparation score [25]. In case of inadequate bowel preparation after fecal residue and fluid suction, the colonoscopy was interrupted and rescheduled. The withdrawal time was measured by an endoscopy-nurse. The net withdrawal time (time of mucosal inspection) was set at a minimum of 6 minutes. All detected lesions were immediately resected and obtained for histologic assessment. When resection of detected lesions was not directly possible, biopsies were taken to provide a histopathologic diagnosis. Polyp characteristics (size, location, morphology and optical diagnosis) were obtained for all lesions.

\section{Pathology}

All tissue specimens were assessed according to the Vienna criteria by one of two expert gastrointestinal pathologists, one in each center [26]. The diagnosis was based on the morphologic features on hematoxylin and eosin staining. Lesions were classified as either adenomatous, serrated, or carcinoma. Adenomatous lesions were subdivided based on the grade of dysplasia (low-grade or high-grade) as well as the presence of a villous component (tub- 


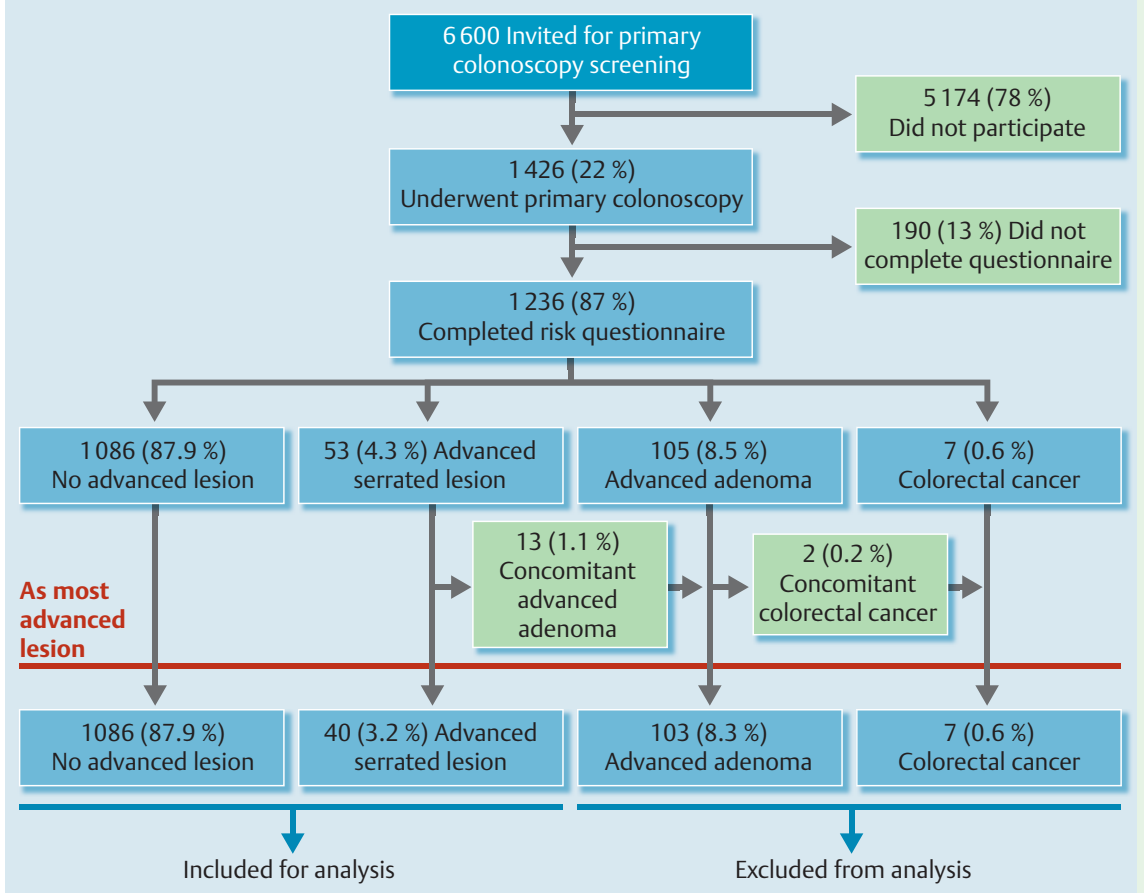

Fig. 1 Study flowchart

ular, tubulovillous or villous). Serrated polyps (SP) were subdivided into hyperplastic polyps (HPs), sessile serrated adenomas/ polyps (SSA/Ps) without dysplasia, SSA/Ps with dysplasia or traditional serrated adenomas (TSAs). A SSA/P was predominantly defined based on the presence of (hyper-)serration up to the base of the crypts as well as distortion of the crypts, presenting with abnormal shapes like the common L-shape or inverted T-shape [11]. A TSA was predominantly defined based on the presence of a complex and distorted tubulovillous or villous configuration, prominent serration, aberrant crypt formation and diffuse cytoplasmic eosinophilia [11]. Advanced adenomas were defined as all adenomas $\geq 10 \mathrm{~mm}$, with a tubulovillous or villous histology and/or with high-grade dysplasia. Advanced neoplasia was defined as either CRC or advanced adenoma. The pathologist of the other center revised all lesions that were primarily classified as advanced neoplasia, as well as a random sample of $10 \%$ of the other lesions. This demonstrated no structural discrepancies between both pathologists. In case of inconsistency, the slides were evaluated by both pathologists together to obtain a consistent diagnosis. Advanced SPs were not reevaluated for the purpose of this study.

\section{Statistical analysis}

We evaluated associations between the putative CRC risk factors and the presence of advanced subtypes of SP. An advanced SP was defined as either a SP $\geq 10 \mathrm{~mm}$ or a SP with (any) dysplasia, based on the European Society of Gastrointestinal Endoscopy (ESGE) post-polypectomy colonoscopy surveillance guideline [17]. We focused on the most advanced lesion per participant in the analysis. Advanced adenomas and CRC were considered as lesions more advanced than advanced SP, while non-advanced adenomas were classified as less advanced. To evaluate risk factors for SP, we compared participants with advanced SP, but without more advanced lesions, with the group of participants without lesions or with non-advanced adenoma and/or non-advanced SP as most advanced lesion.
For each risk factor, the univariate association with the presence of at least one advanced SP was calculated and presented as odds ratio (OR) with 95\% confidence interval (CI). Multivariable logistic regression was used to evaluate the conditional, adjusted associations between the risk factors and the presence of at least one advanced SP. Stepwise backward elimination was used to select risk factors included in the multivariate analysis. All risk factors that showed a $P$ value of $<0.2$ in the univariate analysis were included in the multivariable analysis, as well as gender and age. Multiple imputation was used to handle the missing values from the individual covariates [27].

\section{Results \\ $\nabla$}

\section{Participants}

Of the 6600 invitees, 1426 participated in this trial, and 1236 also completed the risk questionnaire. CRC was detected in seven participants, advanced adenoma in 105, and advanced SP in 53; 1086 participants did not have an advanced lesion. Thirteen participants with an advanced SP also had an advanced adenoma detected, resulting in 40 participants with an advanced SP as most advanced detected lesion. Those 40 participants were included in the analysis, and compared with the 1086 participants who did not have an advanced lesion ( $\bullet$ Fig.1). Table 1 summarizes key characteristics of these 1,126 participants. The mean age was 60.1 years (SD 6.2); 575 were male ( $51 \%$ ).

Of the 40 participants with at least one advanced SP, 26 (65\%) had at least one large SP detected, and 16 (40\%) individuals had at least one SP containing any dysplasia. In one (2.5\%) participant an SP containing high-grade dysplasia was detected. The median number of detected SPs of any kind was two (range 1-8), while in total 14 (35\%) out of these 40 participants were also found to have a non-advanced adenoma (median 0; range $0-3$ ).

In the 1,086 participants without an advanced lesion, there were $249(23 \%)$ in whom at least one non-advanced SP was detected; 
Oryna

Table 1 Diagnostic risk factors for advanced serrated lesions as most advanced lesion in an average-risk screening population

\begin{tabular}{|c|c|c|c|c|c|c|c|}
\hline & Overall & $\begin{array}{l}\text { No advanced } \\
\text { lesion }\end{array}$ & $\begin{array}{l}\text { Advanced } \\
\text { SP }\end{array}$ & $\begin{array}{l}\text { Univariate OR } \\
(95 \% \mathrm{Cl})\end{array}$ & $P$ value & $\begin{array}{l}\text { Multivariable OR } \\
(95 \% \mathrm{Cl})\end{array}$ & $P$ value \\
\hline Cohort (n) & 1126 & 1086 & 40 & & & & \\
\hline Sex & & & & $1.18(0.63-2.25)$ & 0.61 & $1.16(0.61-2.24)$ & 0.65 \\
\hline Male, n (\%) & $575(51.1)$ & $553(50.9)$ & $22(55.0)$ & & & & \\
\hline Female, n (\%) & 551 (48.9) & $533(49.1)$ & $18(45.0)$ & & & & \\
\hline Age in years, mean $(S D)^{1}$ & $60.1(6.2)$ & $60.1(6.2)$ & $60.4(6.6)$ & $1.01(0.96-1.06)$ & 0.71 & $1.02(0.97-1.08)$ & 0.46 \\
\hline$\leq 60, \mathrm{n}(\%)$ & $595(52.8)$ & $572(52.7)$ & $23(57.5)$ & & & & \\
\hline$>60, \mathrm{n}(\%)$ & $531(47.2)$ & $514(47.3)$ & $17(42.5)$ & & & & \\
\hline Smoking status & & & & $3.50(1.74-6.80)$ & $<0.001$ & $4.50(2.23-8.89)$ & $<0.001$ \\
\hline Current smoker, n (\%) & $162(14.4)$ & $148(13.6)$ & $14(35.0)$ & & & & \\
\hline Former/no smoker, n (\%) & $951(84.4)$ & $926(85.3)$ & $25(62.5)$ & & & & \\
\hline Missing, $n(\%)$ & $13(1.2)$ & $12(1.1)$ & $1(2.5)$ & & & & \\
\hline Fiber intake in g/day, mean (SD) ${ }^{1}$ & $41.4(21.8)$ & $41.2(21.7)$ & $47.5(24.5)$ & $1.01(1.00-1.02)$ & 0.07 & $1.02(1.00-1.03)$ & 0.01 \\
\hline$\leq 41, \mathrm{n}(\%)$ & $665(59.1)$ & $646(59.5)$ & $19(47.5)$ & & & & \\
\hline$>41, \mathrm{n}(\%)$ & 461 (41.9) & $440(40.5)$ & $21(52.5)$ & & & & \\
\hline $\mathrm{BMI}$ in $\mathrm{kg} / \mathrm{m}^{2}$, mean $(\mathrm{SD})^{1}$ & $26.6(4.1)$ & $26.6(4.1)$ & $27.6(4.3)$ & $1.06(0.98-1.13)$ & 0.11 & $1.07(0.99-1.15)$ & 0.06 \\
\hline$<25, \mathrm{n}(\%)$ & $417(37.0)$ & $404(37.2)$ & $13(32.5)$ & & & & \\
\hline $25-30, n(\%)$ & $506(44.9)$ & $489(45.0)$ & $17(42.5)$ & & & & \\
\hline$>30, \mathrm{n}(\%)$ & $185(16.4)$ & $175(16.1)$ & $10(25.0)$ & & & & \\
\hline Missing, $\mathrm{n}(\%)$ & $18(1.7)$ & $18(1.7)$ & & & & & \\
\hline \multicolumn{8}{|l|}{ Alcohol in units/week, median } \\
\hline$(I Q R)^{1}$ & $5(1-10)$ & $5(1-10)$ & $4(1-14)$ & $1.01(0.97-1.03)$ & 0.78 & & \\
\hline$\leq 15, \mathrm{n}(\%)$ & $934(82.9)$ & $902(83.0)$ & $32(80.0)$ & & & & \\
\hline$>15, \mathrm{n}(\%)$ & $136(12.1)$ & $129(11.9)$ & $7(17.5)$ & & & & \\
\hline Missing, $\mathrm{n}(\%)$ & $56(5.0)$ & $55(5.1)$ & $1(2.5)$ & & & & \\
\hline \multicolumn{8}{|l|}{ No. of relatives with CRC, median } \\
\hline$(\mathrm{IQR})^{1}$ & $0(0-0)$ & $0(0-0)$ & $0(0-0)$ & $1.15(0.55-2.02)$ & 0.67 & & \\
\hline $0, \mathrm{n}(\%)$ & $964(85.6)$ & $932(85.8)$ & $32(80.0)$ & & & & \\
\hline $1, n(\%)$ & $138(12.3)$ & $130(12.0)$ & $8(20.0)$ & & & & \\
\hline$>1, \mathrm{n}(\%)$ & $24(2.1)$ & $24(2.2)$ & 0 & & & & \\
\hline Intensive exercise & & & & $1.07(0.55-2.02)$ & 0.84 & & \\
\hline$<1$ hour/week, n (\%) & $693(61.5)$ & $669(61.6)$ & $24(60.0)$ & & & & \\
\hline$\leq 1$ hour/week, $\mathrm{n}(\%)$ & $433(38.5)$ & $417(38.4)$ & $16(40.0)$ & & & & \\
\hline Sleep behavior in h/day, mean (SD) ${ }^{1}$ & $7.3(1.1)$ & $7.3(1.1)$ & $7.3(1.0)$ & $1.04(0.80-1.41)$ & 0.78 & & \\
\hline$\leq 7, \mathrm{n}(\%)$ & $597(53.0)$ & $576(53.0)$ & $21(52.5)$ & & & & \\
\hline$>7, n(\%)$ & $463(41.1)$ & $446(41.1)$ & $17(42.5)$ & & & & \\
\hline Missing & $66(5.9)$ & $64(5.9)$ & $2(5.0)$ & & & & \\
\hline NSAID/Aspirin & & & & $0.89(0.38-1.87)$ & 0.77 & & \\
\hline Non-user & $880(78.2)$ & $848(78.1)$ & $32(80.0)$ & & & & \\
\hline User & $246(21.8)$ & $238(21.9)$ & $8(20.0)$ & & & & \\
\hline \multicolumn{8}{|l|}{ Calcium intake in $\mathrm{mg} /$ day, median } \\
\hline$(\mathrm{IQR})^{1}$ & $750(505-960)$ & $750(510-960)$ & $750(486-909)$ & $1.00(1.00-1.00)$ & 0.63 & & \\
\hline$\leq 1200$ & $992(88.1)$ & $957(88.1)$ & $35(87.5)$ & & & & \\
\hline$>1200$ & 134 (11.9) & $129(11.9)$ & $5(12.5)$ & & & & \\
\hline \multicolumn{8}{|l|}{ Red meat in units/week, median } \\
\hline$(\mathrm{IQR})^{1}$ & $3(1-4)$ & $3(1-4)$ & $2(2-4)$ & $1.01(0.85-1.21)$ & 0.91 & & \\
\hline$\leq 3$ & $705(62.6)$ & $681(62.7)$ & $24(60.0)$ & & & & \\
\hline$>3$ & $380(33.7)$ & $365(33.6)$ & $15(37.5)$ & & & & \\
\hline Missing & $41(3.7)$ & $40(3.7)$ & $1(2.5)$ & & & & \\
\hline FIT value in $\mathrm{ng} / \mathrm{ml}$, median $(\mathrm{IQR})^{1}$ & $0(0-5)$ & $0(0-5)$ & $0(0-11)$ & $1.00(1.00-1.00)$ & 0.99 & & \\
\hline$\leq 50$ & $943(83.7)$ & $912(84.0)$ & $31(77.5)$ & & & & \\
\hline$>50$ & $65(5.8)$ & $61(5.6)$ & $4(10.0)$ & & & & \\
\hline Missing & $118(10.5)$ & $113(10.4)$ & $5(12.5)$ & & & & \\
\hline
\end{tabular}

BMI, body mass index; CRC, colorectal cancer; NSAID, nonsteroidal anti-inflammatory drug; FIT, fecal immunochemical test; IQR, interquartile range

${ }^{1}$ Quantitative variables were treated as such in the analyses

$21(1.9 \%)$ had at least one SSA/P. There were 243 (22\%) participants with at least one non-advanced adenoma.

\section{CRC risk factors}

In 1 Table1 associations between the CRC risk factors and the presence of at least one advanced SP as most advanced lesion are presented. In univariate analysis we observed a strong association between current smoking and the presence of an ad- vanced SP as most advanced lesion (OR 3.50; (95\% CI 1.74-6.80; $P<0.001$ ). In addition, higher fiber intake (OR 1.24 per 20 gram; $95 \% \mathrm{CI} 0.98-1.58 ; P=0.07)$ as well as higher body mass index (BMI) (OR 1.06 per point; $95 \% \mathrm{Cl} 0.98-1.13 ; P=0.11$ ) also showed a moderate association, although this did not reach statistical significance. Other clinical risk factors were not associated with the presence of an advanced SP as most advanced lesion. This also included fecal hemoglobin concentration (OR 1.00 per $10 \mathrm{ng} / \mathrm{mL}$; 
95\% CI 0.97-1.03, $P=0.99$ ). The association between current smoking and the presence of an advanced SP as most advanced lesion remained significant in the multivariable analysis.(OR 4.50; $95 \%$ CI $2.23-8.89 ; P<0.001)$. A significant association was also demonstrated for higher fiber intake (OR 1.36 per 20 -g intake; CI 1.07-1.73; $P=0.01$ ). Higher BMI was not significantly associated with advanced SPs in the multivariable analysis (OR 1.07 per point; CI $0.99-1.15 ; P=0.06$ ).

\section{Discussion}

Our analysis shows that current smoking is strongly associated with advanced SPs as most advanced detected lesion in an asymptomatic screening population, with an odds ratio of about four. In addition, higher fiber intake was moderately associated with the presence of advanced SPs. Other CRC risk factors, including fecal hemoglobin level, did not show a significant association with the presence of at least one advanced SP.

All available data for this analysis had been prospectively collected in a structured and transparent manner. All colonoscopies were performed according to the most up-to-date colonoscopy quality parameters and endoscopists were instructed to resect all detected lesions, unaware of location or predicted histology [19]. Yet a number of potential limitations have to be acknowledged. The number of subjects with at least one advanced SP was relatively small, which limits the power to detect more moderate associations. For this reason we decided not to perform any sub-analyses in this study. In a recent study we described the prevalence of SP subtypes in the same cohort, showing that the overall detection rate of HPs as well as SSA/Ps was sufficient and comparable to other recent reports [28]. Therefore, the low number of advanced SP, as reported in the current study, most probably is a realistic representation of the prevalence of disease in average-risk individuals. Associations between clinical risk factors and advanced SPs were based on a self-completed questionnaire, which has the risk of socially desirable answers. To limit this effect, participants were told that the results from the questionnaire would be anonymized and in no way affect options for treatment.

A number of previous studies have assessed the association between smoking behavior and the occurrence of SPs, all reporting a moderate to strong association [29-37]. However, most studies had a case-control design, were executed in an era during which the risk of SPs was not yet well defined and/or were not stratified for SP subtype [29-33,35,37]. Although well designed, the risk information value of smoking behavior for the detection of advanced SPs in an average-risk asymptomatic screening population, therefore, could not be reliably assessed in these studies. Two recent studies evaluated the association between smoking and SPs via a prospective cohort design $[34,36]$. The first study, performed in a cohort of 985 asymptomatic, screening-naïve participants, reported a significant association between current smoking and the prevalence of SSA/Ps, with an odds ratio of about five [34]. Male gender and increasing age were also associated with the presence of SSA/Ps in this study. However, the detection rate of SSA/Ps was reported to be only $2.3 \%$. Because these associations were not adjusted for concomitant advanced adenomas, they are difficult to interpret. In the second study a combined analysis of three colonoscopy-based clinical trials $(n=$ 2915 patients) was conducted to evaluate the smoking-associated risk of adenomas as well as serrated polyps compared to patients without any polyps [36]. Smoking appeared to be associated with greater risk of serrated polyps, particularly in the leftsided colon. Sub-analysis by polyp size showed a moderate association between smoking behavior and large SPs (current smoking: RR 1.62, 95\% CI 1.27 - 2.07 and former smoking: RR 1.21, 95\% CI $0.98-1.49)$. This association was far more moderate than found in the current study. The causal pathway between smoking and SPs is still under debate. One recent study has shown that tobacco smoking leads to extensive genome-wide changes in DNA methylation, which, to a large extent, returns to the level of nonsmokers when discontinued [38]. This would explain the increased risk for current smokers compared to former smokers and non-smokers, as found in our study. Unfortunately, sub-analysis for the risk difference between former smokers and nonsmokers could not be performed due to the restrictions in sample size.

Studies that have assessed the relationship between fiber intake and the prevalence of SPs are scarce [33]. In a large colonoscopybased case-control study, a protective trend of higher fiber intake was found for the detection of SPs, which seems contrary to our findings [33]. In that study, all participants with at least one SP were included in the analysis, regardless of SP subtype. This means that diminutive HPs in the rectosigmoid were also included, which makes an informative comparison of the results difficult. The role of fiber intake in the pathogenesis of SPs, if any, is still speculative. It has been suggested that the development of advanced lesions through the serrated neoplasia pathway is induced by alterations in the microbiome of these participants [39]. Fiber intake may have a role in this process.

Two other recent studies have assessed the diagnostic accuracy of FIT for the detection of SPs larger than $1 \mathrm{~cm}[10,18]$. These showed that including large SPs in the target condition leads to a decreased diagnostic performance of FIT. In a large study comparing FIT-based screening to multitarget stool DNA testing (including the quantitative molecular assays for KRAS mutations, aberrant NDRG4 and BMP3 methylation, $\beta$-actin and fecal haemoglobin levels) for CRC screening, only $5.1 \%$ of participants with a large SP had a quantitative FIT-score above $100 \mathrm{ng}$ hemoglobin per $\mathrm{mL}$ of buffer, while $42.4 \%$ of participants were detected with DNA testing, at the same specificity level [10]. In another study, the diagnostic performance of BMP3 methylation alone was compared to FIT for the detection of SPs larger than $1 \mathrm{~cm}$, showing that at $95 \%$ specificity, the stool assay of BMP3 methylation detected $63 \%$ of large SPs, compared to $0 \%$ by FIT [18].

In our analysis, the performance of fecal hemoglobin in detecting advanced SPs was very limited. At a cut-off of $50 \mathrm{ng} / \mathrm{mL}$, the sensitivity for the detection of advanced SPs in those without other, more advanced lesions, was $11 \%$ at a specificity of $94 \%$. At a cutoff of $100 \mathrm{ng} / \mathrm{mL}$, sensitivity would be $5.7 \%$ with a specificity of $97 \%$. To put this in perspective: the sensitivity of current smoking, considered in isolation, was $36 \%$ for a specificity of $86 \%$.

We believe that the low performance of FIT in detecting advanced adenoma and advanced SPs, and the promising information of CRC risk factors, could and should lead to the development of better-performing population screening programs. These would be programs that not only detect CRC, or advanced adenoma, but also advanced SPs. Such programs may include fecal hemoglobin, DNA testing, and other markers, but they should not ignore the incremental value of easy-to-collect information on CRC risk factors, such as smoking behavior. Ideally, the value of fecal hemoglobin levels, other fecal DNA markers and clinical risk factors such as smoking status will be used in a risk stratification model 
in which the individual fitted risk value determines the need for a subsequent colonoscopy. Future research should focus on the validation of such a model.

\section{Competing interests: None}

\section{Institutions}

${ }^{1}$ Department of Gastroenterology and Hepatology, Academic Medical Centre, University of Amsterdam, Amsterdam, Netherlands

${ }^{2}$ Department of Clinical Epidemiology, Biostatistics and Bioinformatics, Academic Medical Center, Amsterdam, Netherlands

${ }^{3}$ Department of Gastroenterology and Hepatology, Erasmus MC University Medical Centre, Rotterdam, Netherlands

${ }^{4}$ Department of Otolaryngology, University Medical Center Utrecht, 3508 GA Utrecht, The Netherlands

Department of Gastroenterology and Hepatology, National Cancer Institute, Amsterdam, The Netherlands

\section{References}

1 Zauber AAG, Winawer SSJ, O'Brien MJ et al. Colonoscopic polypectomy and long-term prevention of colorectal-cancer deaths. N Engl J Med 2012; 67: 355-356

2 Løberg M, Kalager M, Holme Ø et al. Long-term colorectal-cancer mortality after adenoma removal. N Engl J Med 2014; 371: 799-807

3 Lansdorp-Vogelaar I, Knudsen AB, Brenner H. Cost-effectiveness of colorectal cancer screening. Epidemiol Rev 2011; 33: 88-100

4 Rex DK, Johnson DA, Anderson JC et al. American College of Gastroenterology guidelines for colorectal cancer screening 2009 [corrected]. Am J Gastroenterol 2009; 104: 739-750

5 Van Rossum LGM, van Rijn AF, Verbeek ALM et al. Colorectal cancer screening comparing no screening, immunochemical and guaiac fecal occult blood tests: a cost-effectiveness analysis. Int J Cancer 2011; 128 : $1908-1917$

6 Parente F, Boemo C, Ardizzoia A et al. Outcomes and cost evaluation of the first two rounds of a colorectal cancer screening program based on immunochemical fecal occult blood test in northern Italy. Endoscopy 2013; 45: $27-34$

7 Kapidzic A, Grobbee EJ, Hol L et al. Attendance and Yield Over Three Rounds of Population-Based Fecal Immunochemical Test Screening. Am J Gastroenterol 2014; 8: 1-9

8 De Wijkerslooth TR, Stoop EM, Bossuyt PM et al. Immunochemical fecal occult blood testing is equally sensitive for proximal and distal advanced neoplasia. Am J Gastroenterol 2012; 107: 1570-1578

9 Stegeman I, de Wijkerslooth TR, Stoop EM et al. Combining risk factors with faecal immunochemical test outcome for selecting CRC screenees for colonoscopy. Gut 2014; 63: 466-471

10 Imperiale TF, Ransohoff DF, Itzkowitz SH et al. Multitarget stool DNA testing for colorectal-cancer screening. N Engl J Med 2014; 370: 1287-97

11 Snover DC, Ahnen DJ, Burt RW et al. Serrated polyps of the colon and rectum and serrated polyposis. In: Bosman T, Carneiro F, Hruban R, et al. WHO Classification of Tumours of the digestive system. Lyon: 2010: $160-165$

12 Rex DK, Ahnen DJ, Baron JA et al. Serrated lesions of the colorectum: review and recommendations from an expert panel. Am J Gastroenterol 2012; 107: 1315 - 1329 quiz 1314, 1330

13 Rosty C, Hewett DG, Brown IS et al. Serrated polyps of the large intestine: current understanding of diagnosis, pathogenesis, and clinical management. J Gastroenterol 2013; 48: 287-302

14 Snover $D C$. Update on the serrated pathway to colorectal carcinoma. Hum Pathol 2011; 42: 1-10

15 Arain MA, Sawhney M, Sheikh S et al. CIMP status of interval colon cancers: another piece to the puzzle. Am J Gastroenterol 2010; 105: $1189-1195$

16 Nishihara $R, W u$ K, Lochhead P et al. Long-term colorectal-cancer incidence and mortality after lower endoscopy. N Engl J Med 2013; 369: $1095-1105$
17 Hassan C, Quintero E, Dumonceau J-M et al. Post-polypectomy colonoscopy surveillance: European Society of Gastrointestinal Endoscopy (ESGE) Guideline. Endoscopy 2013; 45: 842 - 851

18 Heigh RI, Yab TC, Taylor WR et al. Detection of colorectal serrated polyps by stool DNA testing: comparison with fecal immunochemical testing for occult blood (FIT). PLoS One 2014; 9: e85659

19 De Wijkerslooth TR, de Haan MC, Stoop EM et al. Study protocol: population screening for colorectal cancer by colonoscopy or CT colonography: a randomized controlled trial. BMC Gastroenterol 2010; 10: 47

20 Stegeman I, de Wijkerslooth TR, Stoop EM et al. Colorectal cancer risk factors in the detection of advanced adenoma and colorectal cancer. Cancer Epidemiol 2013; 37: 278 -283

21 Colkesen EB, Ferket BS, Tijssen JGP et al. Effects on cardiovascular disease risk of a web-based health risk assessment with tailored health advice: A follow-up study. Vasc Health Risk Manag 2011; 7: 67-74

22 Van den Brink C, Ocke M, Houben A et al. Validering van standaardvraagstelling voeding voor Lokale en Nationale Monitor Volksgezondheid (validation of a community health services food consumption questionnaire in the Netherlands). RIVM Rapp 2608540082005 : 1 43

23 Yusuf PS, Hawken S, Ônpuu S et al. Effect of potentially modifiable risk factors associated with myocardial infarction in 52 countries (the INTERHEART study): Case-control study. Lancet 2004; 364: 937-952

24 Rex DK, Petrini JL, Baron TH et al. Quality indicators for colonoscopy. Am J Gastroenterol 2006; 101: 873-85

25 Rostom A, Jolicoeur E. Validation of a new scale for the assessment of bowel preparation quality. Gastrointest Endosc 2004; 59: 482 - 486

26 Schlemper RJ. The Vienna classification of gastrointestinal epithelial neoplasia. Gut 2000; 47: 251 - 255

27 Streiner $D L$. The case of the missing data: Methods of dealing with dropouts and other research vagaries. Can J Psychiatry 2002; 47: 68 75

28 Hazewinkel Y, de Wijkerslooth TR, Stoop EM et al. Prevalence of serrated polyps and association with synchronous advanced neoplasia in screening colonoscopy. Endoscopy 2014; 46: 219-224

29 Lieberman DA, Prindiville S, Weiss DG et al. Risk factors for advanced colonic neoplasia and hyperplastic polyps in asymptomatic individuals. JAMA 2003; 290: 2959-2967

30 Morimoto LM, Newcomb PA, Ulrich CM et al. Risk factors for hyperplastic and adenomatous polyps: Evidence for malignant potential? Cancer Epidemiol Biomarkers Prev 2002; 11: 1012-1018

31 Burnett-Hartman AN, Newcomb PA, Potter JD et al. Genomic aberrations occurring in subsets of serrated colorectal lesions but not conventional adenomas. Cancer Res 2013; 73: 2863 - 2872

32 Ji BT, Weissfeld JL, Chow WH et al. Tobacco smoking and colorectal hyperplastic and adenomatous polyps. Cancer Epidemiol Biomarkers Prev 2006; 15: 897-901

$33 \mathrm{Fu} Z$, Shrubsole MJ, Smalley WE et al. Lifestyle factors and their combined impact on the risk of colorectal polyps. Am J Epidemiol 2012; 176: $766-776$

34 Buda A, De Bona M, Dotti I et al. Prevalence of different subtypes of serrated polyps and risk of synchronous advanced colorectal neoplasia in average-risk population undergoing first-time colonoscopy. Clin Transl Gastroenterol 2012; 3: e6

35 Anderson JC, Rangasamy P, Rustagi $T$ et al. Risk factors for sessile serrated adenomas. J Clin Gastroenterol 2011; 45: 694-699

36 Figueiredo JC, Crockett SD, Snover DC et al. Smoking-associated risks of conventional adenomas and serrated polyps in the colorectum. Cancer Causes Control 2014; 26: 377-386

37 Rustagi T, Rangasamy P, Myers M et al. Sessile serrated adenomas in the proximal colon are likely to be flat, large and occur in smokers. World J Gastroenterol 2013; 19: 5271 - 5277

38 Zeilinger S, Kühnel B, Klopp $N$ et al. Tobacco smoking leads to extensive genome-wide changes in DNA methylation. PLoS One 2013; 8: e63812

39 Tahara T, Yamamoto E, Suzuki $\mathrm{H}$ et al. Fusobacterium in colonic flora and molecular features of colorectal carcinoma. Cancer Res 2014; 74: 1311-1318 